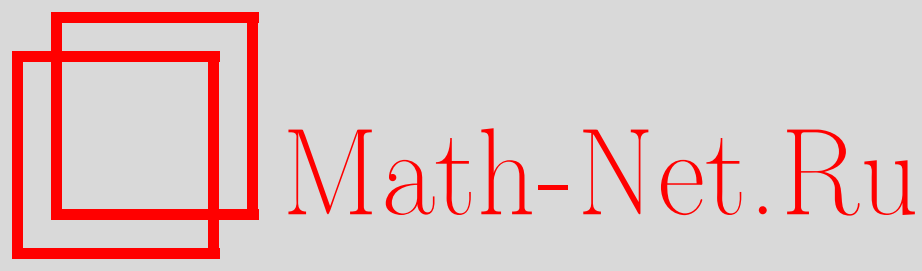

А. А. Андрианов, В. А. Андрианов, Д. Эсприу, С. С. Колеватов, Влияние градиента псевдоскалярного конденсата на режим остывания компактных звезд, ТМФ, 2017, том 190, номер 2, 207-225

DOI: https://doi.org/10.4213/tmf9134

Использование Общероссийского математического портала Math-Net.Ru подразумевает, что вы прочитали и согласны с пользовательским соглашением http: //www.mathnet.ru/rus/agreement

Параметры загрузки:

IP : 52.87 .193 .239

26 апреля 2023 г., 14:10:54

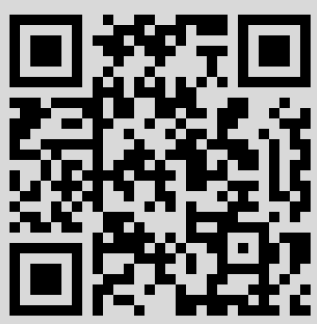




\title{
ФИЗИКА
}

Том 190, № 2

февраль, 2017

(C) 2017 г.

А. А. Андрианов ${ }^{* \dagger}$, В. А. Андрианов*, Д. Эсприу ${ }^{\dagger}$, С. С. Колеватов*

ВЛИЯНИЕ ГРАДИЕНТА

\section{ПСЕВДОСКАЛЯРНОГО КОНДЕНСАТА}

\section{НА РЕЖИМ ОСТЫВАНИЯ КОМПАКТНЫХ ЗВЕЗД}

\begin{abstract}
Рассматриваются процессы в компактных звездах, возникающие при возможном образовании псевдоскалярного конденсата в конечных объемах. В paботе не делается конкретных предположений о природе конденсата. В предположении, что в областях с изменяющейся псевдоскалярной плотностью распространение фотонов можно описать в рамках электродинамики Максвелла-Черна-Саймонса, найдены коэффициенты отражения/прохождения для областей с разными плотностями. Проведено исследование фермионного спектра в присутствии аксиального поля с учетом градиента псевдоскалярного конденсата, а также исследовано влияние модифицированных спектров фотонов и фермионов на процесс остывания компактных звезд.
\end{abstract}

Ключевые слова: компактные звезды, псевдоскалярный конденсат, электродинамика Максвелла-Черна-Саймонса, граничные эффекты.

DOI: $10.4213 / \operatorname{tmf9134}$

Работа поддержана грантами FPA2013-46570, 2014-SGR-104 и Consolider CPAN. Работа также частично поддержана Ministerio de Economía, Industria у Competitividad (MINECO) (проект MDM-2014-0369). Работа А. А. Андрианова, В. А. Андрианова и С. С. Колеватова финансировалась РФФИ (грант № 16-02-00348). Работа А. А. Андрианова также поддержана Санкт-Петербургским государственным университетом (грант № 11.42.1049.2016). Работа В. А. Андрианова поддержана Санкт-Петербургским государственным университетом (грант № 11.42.1051.2016). Работа С. С. Колеватова поддержана Санкт-Петербургским государственным университетом (грант № 11.41.821.2015). С. С. Колеватов также благодарен фонду “Династия".

* Санкт-Петербургский государственный университет, Санкт-Петербург, Россия. E-mail: andrianov@icc.ub.edu,kss2005@list.ru

${ }^{\dagger}$ Departament d'Estructura i Constituents de la Matèria and Institut de Ciències del Cosmos, Universitat de Barcelona Martí i Franquès 1, Barcelona, Spain. 


\section{1. ВВЕДЕНИЕ}

Интерес к возможным нарушениям симметрии Лоренца и других фундаментальных симметрий значительно возрос после появления работы [1], в которой была рассмотрена электродинамика с дополнительным членом Черна-Саймонса (ЧС), ориентированным вдоль постоянного 4-вектора ЧС. В этой же работе обсуждалась возможность наблюдаемости некоторых эффектов, связанных с нарушением пространственной четности, в излучении далеких радиогалактик и было показано, что соответствующие эффекты не наблюдаются. Однако все же есть некоторые области, в которых электродинамика Максвелла-Черна-Саймонса (МЧС, также известная как электродинамика Кэрролла-Филда-Джекива) может описывать происходящие там процессы.

В работе [2] было сделано предположение, что нарушение пространственной четности может возникать при нецентральных столкновениях тяжелых ионов, проявляясь в виде кирального магнитного эффекта [3]. Спонтанное нарушение пространственной четности также может возникать при достаточно большой плотности барионного вещества [4]. Не так давно несколько экспериментов по столкновению тяжелых ионов выявили аномальный выход лептонных пар [5], [6], и было показано, что данный эффект может быть вызван локальным нарушением пространственной четности в сталкивающихся ядрах [7], [8]. Этот эффект возникает благодаря взаимодействию топологических флуктуаций в КХД [9], [10] с электродинамикой МЧС.

Другой возможный источник макроскопического нарушения пространственной четности - аксионный фон. До сих пор не было получено никаких экспериментальных доказательств существования аксионов, однако теоретически было показано [11]-[14], что нарушение пространственной четности может возникать при бозеэйнштейновской конденсации аксионов или аксионоподобных полей на различных астрофизических масштабах. В частности, это может происходить внутри звезд [15]. Подобные аксионные конденсаты могут оказаться достаточно компактными [16]. В плотной ядерной среде следует также учесть явления, связанные с пионным [17] и киральным конденсатом [18]. В настоящей работе не рассматривается природа образования псевдоскалярного конденсата, нам важна лишь величина градиента его плотности в среде. Дело в том, что для области с постоянной плотностью псевдоскалярного конденсата вектор ЧС обращается в ноль и электромагнитные свойства можно описать обычной максвелловской электродинамикой. То же поведение верно и для постоянного аксиально-векторного взаимодействия с фермионами [19], [20].

В последующих разделах мы рассматриваем эффекты, связанные с появлением псевдоскалярного градиента в плотной среде. Для начала мы обсуждаем, как меняется кинематика фотонов и фермионов в интересующей нас области, и показываем, что изменения отличаются для различных поляризаций фотонов, а в случае фермионов море Ферми расщепляется на две части с различающимися уровнями Ферми. Затем мы выписываем коэффициенты отражения и прохождения для фотонов, пересекающих границу областей с разными уравнениями состояния, и показываем, что эффекты отражения могут значительно влиять на процесс радиационного охлаждения, если градиент плотности больше характерной температуры среды. Подобный процесс происходит и для фермионов, однако эффекты, связанные с фермионами, значительно подавлены эффективной фермионной массой. Фермио- 


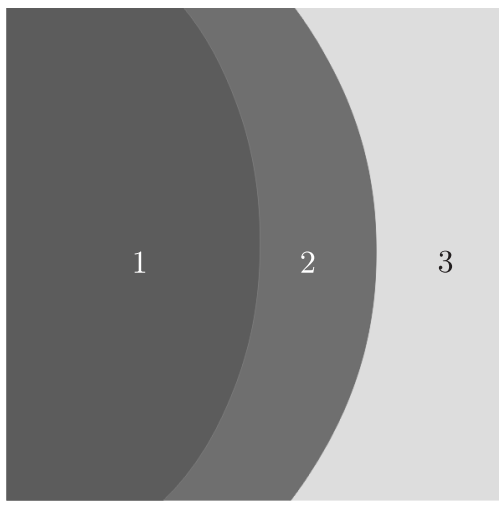

Рис. 1. Границы доменов.

ны могут "распадаться" в среде, испуская фотон. Более того, фотоны с определенной поляризацией, распространяющиеся в описываемой среде, могут распадаться на фермион-антифермионную пару. В заключении мы обсуждаем предположения относительно применимости описываемых нами явлений в вырожденных фермионных системах, например в плотных звездах, где возможно образование псевдоскалярного конденсата.

\section{2. СПЕКТР ФОТОНОВ В ПРИСУТСТВИИ ГРАДИЕНТА ПСЕВДОСКАЛЯРНОГО КОНДЕНСАТА}

Предположим, что в описываемой нами плотной системе присутствуют домены с различными значениями псевдоскалярного конденсата. Каждому домену соответствует своя величина конденсата $a_{i}, a=0$ также является допустимым значением. Следует ожидать, что между различными доменами существует переходная область. Рис. 1 иллюстрирует общую ситуацию: область 2 есть переходная область с ненулевым градиентом конденсата, разделяющая области 1 и 3.

В переходной области поведение фотонов можно описать с помощью модели Кэрролла-Филда-Джекива (или электродинамикой МЧС) с плотностью лагранжиана

$$
\mathcal{L}=-\frac{1}{4} F^{\alpha \beta}(x) F_{\alpha \beta}(x)-\frac{1}{4} F^{\mu \nu}(x) \widetilde{F}_{\mu \nu}(x) a(x),
$$

где $A_{\mu}(x)$ и $a(x)$ - векторное и эффективное псевдоскалярное поля соответственно, $\widetilde{F}^{\mu \nu}=(1 / 2) \varepsilon^{\mu \nu \rho \sigma} F_{\rho \sigma}-$ дуальная напряженность поля.

Псевдоскалярное поле различно для различных областей. Так, в областях 1 и 3 поле $a(x)$ остается почти постоянным и второй член в лагранжиане не дает вклада в уравнения поля, в результате в этих областях можно использовать обычную электродинамику. В области 2 мы предполагаем, что $a$ меняется линейно от $a_{-}$до $a_{+}$. В этом случае псевдоскалярное поле можно локально выразить как

$$
a(x)=\zeta \cdot x\left[\theta\left(\zeta \cdot\left(x-x_{-}\right)\right)-\theta\left(\zeta \cdot\left(x-x_{+}\right)\right)\right], \quad \zeta \cdot x \equiv \zeta_{\lambda} x^{\lambda},
$$

где $\zeta^{\mu}$ - постоянный вектор с размерностью массы, пропорциональный градиенту псевдоскалярного конденсата. Мы предполагаем, что длина волны фотона значительно меньше толщины описываемого слоя, и считаем последнюю бесконечной для 
упрощения наших вычислений. Таким образом, инвариантность Лоренца оказывается нарушенной в полупространстве Минковского.

Рассмотрим небольшой объем в пограничном слое, который не чувствует кривизну границы, выберем для простоты первую пространственную координату вдоль локального радиуса кривизны домена и установим $x_{-}$равным $x_{1}=0$. В этом случае справделиво равенство

$$
a(x)=\zeta x_{1} \theta\left(x_{1}\right),
$$

где мы предположили, что толщина слоя между доменами значительно больше характерной длины волны фотона и положили $x_{+} \rightarrow \infty$. Это предположение позволяет нам значительно упростить уравнения поля:

$$
\square A^{\nu}+\zeta \varepsilon^{1 \nu \sigma \rho} \theta\left(x_{1}\right) \partial_{\sigma} A_{\rho}=0 .
$$

Это уравнение ранее было решено в статье [21], краткое описание решения и вид векторов поляризации для области $x_{1}>0$ можно найти в приложении. В области, в которой действует электродинамика МЧС, соответствующие дисперсионные соотношения выглядят следующим образом:

$$
\omega^{2}= \begin{cases}\mathbf{k}^{2} & \text { для } L \text {-поляризации, } \\ \mathbf{k}^{2}+\zeta^{2} / 2+\zeta \sqrt{k_{1}^{2}+\zeta^{2} / 4} & \text { для “+”-поляризации, } \\ \mathbf{k}^{2}+\zeta^{2} / 2-\zeta \sqrt{k_{1}^{2}+\zeta^{2} / 4} & \text { для “-"-поляризации, }\end{cases}
$$

или в форме, более удобной для расчета прохождения фотонов через границу:

$$
\begin{aligned}
& k_{1 L}^{\mathrm{CS}}=k_{1}^{0}=\sqrt{\omega^{2}-k_{\perp}^{2}}, \\
& k_{1+}^{\mathrm{CS}}=\sqrt{\omega^{2}-k_{\perp}^{2}-\zeta \sqrt{\omega^{2}-k_{\perp}^{2}}}, \\
& k_{1-}^{\mathrm{CS}}=\sqrt{\omega^{2}-k_{\perp}^{2}+\zeta \sqrt{\omega^{2}-k_{\perp}^{2}}} .
\end{aligned}
$$

Здесь индекс "0" обозначает пространство без нарушения пространственной четности, $\omega$ - энергия фотона, $\vec{k}_{\perp}=\left(0, k_{2}, k_{3}\right)$ - поперечный волновой вектор фотона. Это выражение описывает три различные поляризации фотона в области с электродинамикой МЧС, т. е. в области, где $x_{1}>0$. Как и следовало ожидать, обычное максвелловское поведение фотонов можно получить, если $\zeta=0$.

Групповые скорости для различных ветвей дисперсионных законов при пространственноподобном векторе ЧС не превышают единицы (см. статью [19], а также работы [22], [23]).

\section{3. ФЕРМИОННЫЙ СПЕКТР В ПРИСУТСТВИИ ГРАДИЕНТА ПСЕВДОСКАЛЯРНОГО КОНДЕНСАТА}

В этом разделе мы исследуем ключевые свойства спинорных полей в присутствии кинетического члена, нарушающего инвариантность Лоренца и связанного с постоянным аксиальным вектором $b_{\mu}$. Предполагается, что этот вектор появляется в области между двумя доменами с различными значениями псевдоскалярного 
конденсата и его присутствие значительно влияет на свойства фермионов с энергиями, близкими к поверхности Ферми.

Спектр свободных фермионов можно получить из измененного уравнения Дирака в импульсном представлении:

$$
\left(\gamma^{\mu} p_{\mu}-m-\gamma^{\mu} b_{\mu} \gamma_{5}\right) \psi=0 .
$$

Решение данного уравнения было получено ранее в статье [24]. Спектр фермионов определяется следующим выражением:

$$
\left(p^{2}+\tilde{b}^{2}-m^{2}\right)^{2}+4 \tilde{b}^{2} m^{2}-4(\tilde{b} \cdot p)^{2}=0 .
$$

Соответствующие решения модифицированного уравнения Дирака приведены в приложении к настоящей статье. Это уравнение имеет решения для любых значений $b_{\mu} \equiv \tilde{b}$. Однако квантование спинорного поля можно произвести только в случае наличия пары корней (8) с противоположными знаками и массовой щелью между ними. Эти условия выполняются [25] для небольших $b_{\mu}$, а наличие массовой щели дает нам отсутствие решений с $p_{0}=0$. Подобные решения не возникают для пространственноподобного $b_{\mu}$, используемого нами в настоящей работе. Более того, выбор $\vec{b}^{2}<m^{2}$ достаточно разумен для описания величины постоянного аксиального вектора, соответствующего градиенту псевдоскалярного конденсата, поскольку (как показано в следующем разделе) эта величина не должна превышать нескольких КэВ.

Для пространственноподобного случая мы можем привести $b_{\mu}$ к виду

$$
b_{\mu}=(0, \vec{b})=(0, b, 0,0), \quad b>0,
$$

правильным выбором системы координат. Тогда дисперсионный закон определяется следующим образом:

$$
\begin{gathered}
\omega^{2}=\vec{p}_{\perp}^{2}+p_{1}^{2}+b^{2}+m^{2} \pm 2 b \sqrt{p_{1}^{2}+m^{2}}=\vec{p}_{\perp}^{2}+\left(b \pm \sqrt{p_{1}^{2}+m^{2}}\right)^{2} \\
\vec{p}=\left(p_{1}, p_{2}, p_{3}\right), \quad \vec{p}_{\perp}=\left(0, p_{2}, p_{3}\right) .
\end{gathered}
$$

Эти решения разделены конусом устойчивости. Границу устойчивости $p_{\mu}^{2}=0$ можно записать как

$$
\left|p_{1}\right|=\frac{m^{2}-b^{2}}{2 b}
$$

В нерелятивистском приближении (при малых значениях импульса) дисперсионные соотношения для фермионов определяются соотношением

$$
\omega=m \pm b+\frac{p_{1}^{2}}{2 m}+\frac{p_{\perp}^{2}}{2 m} .
$$

Далее предположим, что среда находится в тепловом равновесии с температурой $T$. Две разные массовые поверхности приведут к двум различным морям Ферми. В нерелятивистском пределе они соответствуют “土”-ветвям написанного выше выражения. Соответствующие значения химического потенциала можно получить, решая уравнение относительно $\mu_{ \pm}$для различных чисел заполнения:

$$
N_{ \pm}=V \int d \omega \frac{\sqrt{(2 m)^{3}(\omega \pm b)}}{4 \pi^{2}} \frac{1}{e^{\left(\omega-\mu_{ \pm}\right) / T}+1} .
$$


Это дает нам связь вида $\mu_{+}=\mu_{-}+2 b$ и соответственно неравенство для границ Ферми $\epsilon_{\mathrm{F}}^{+}>\epsilon_{\mathrm{F}}^{-}$(мы предполагаем, что $b$ положительно). Существование областей с ненулевым градиентом псевдоскалярного поля приводит к расщеплению моря Ферми на два набора с разницей между энергетическими уровнями, пропорциональной значению градиента. Для простоты в данной работе мы рассматриваем случай, когда градиент постоянен.

\section{4. РАСПРОСТРАНЕНИЕ ФОТОНОВ В СРЕДЕ}

Рассмотрим распространение фотонов внутри каждой из областей, указанных на рис. 1. Области 1 и 3 соответствуют выбору $\zeta=0$, тогда как для области 2 $\zeta \neq 0$, поскольку имеет место ненулевой градиент псевдоскалярного поля. Как было показано нами ранее, для различных областей имеют место разные дисперсионные соотношения и, как следствие, возникают нетривиальные эффекты отражения и прохождения. Впервые эти эффекты были описаны в работах [21], [26].

4.1. Прохождение в граничный слой. Предположим, что толщина переходного слоя (области 2 на рис. 1) много больше характерной длины волны и длины свободного пробега фотона. Учитывая эти предположения, воспользуемся (4). Задача сводится к рассмотрению фотонов, падающих на границу из области с обычной электродинамикой и частично проникающих в область, описываемую электродинамикой МЧС. Условия сшивки для этой задачи были подробно описаны в статье [21]. Для определения доли фотонов, проходящих границу, напомним полученные результаты. Решение (4) можно найти с использованием преобразования Фурье по всем координатам, кроме $x_{1}$, и записать в следующем виде:

$$
\tilde{A}_{\nu}= \begin{cases}\tilde{u}_{\nu \rightarrow} e^{i k_{1}^{0} x_{1}}+\tilde{u}_{\nu \leftarrow} e^{-i k_{1}^{0} x_{1}}, & x_{1}<0, \\ \sum_{A}\left[\tilde{v}_{\nu A \rightarrow} e^{i k_{1 A}^{\mathrm{CS}} x_{1}}+\tilde{v}_{\nu A \leftarrow} e^{-i k_{1 A}^{\mathrm{CS}} x_{1}}\right], & x_{1}>0 .\end{cases}
$$

Первый индекс у $\tilde{v}$ обозначает соответствующую компоненту вектора $A_{\nu}, \nu=0,2,3$, второй индекс $A$ соответствует поляризации, стрелки указывают на направление распространения частицы. Более того, выполняются следующие равенства:

$$
\begin{aligned}
& \tilde{v}_{2+\rightleftarrows}=\frac{k_{2} k_{3}+i \omega \sqrt{\omega^{2}-k_{\perp}^{2}}}{\omega^{2}-k_{2}^{2}} \tilde{v}_{3+\rightleftarrows}, \\
& \tilde{v}_{2-\rightleftarrows}=\frac{k_{2} k_{3}-i \omega \sqrt{\omega^{2}-k_{\perp}^{2}}}{\omega^{2}-k_{2}^{2}} \tilde{v}_{3-\rightleftarrows}, \\
& \tilde{v}_{0+\rightleftarrows}=-\frac{\omega k_{3}-i k_{2} \sqrt{\omega^{2}-k_{\perp}^{2}}}{2\left(\omega^{2}-k_{\perp}^{2}\right)} \tilde{v}_{3+\rightleftarrows}, \\
& \tilde{v}_{0-\rightleftarrows}=-\frac{\omega k_{3}+i k_{2} \sqrt{\omega^{2}-k_{\perp}^{2}}}{2\left(\omega^{2}-k_{\perp}^{2}\right)} \tilde{v}_{3-\rightleftarrows}, \\
& \tilde{v}_{2 L \rightleftarrows}=\frac{k_{2}}{k_{3}} \tilde{v}_{3 L \rightleftarrows}, \\
& \tilde{v}_{0 L \rightleftarrows}=-\frac{\omega}{k_{3}} \tilde{v}_{3 L \rightleftarrows} \cdot
\end{aligned}
$$




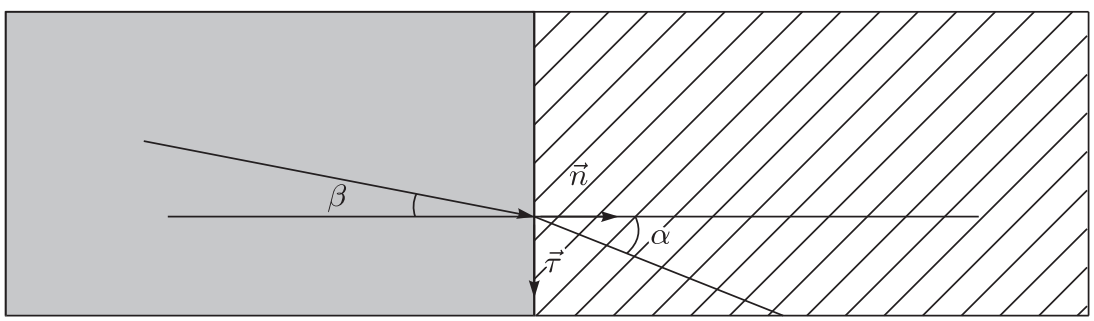

Рис. 2. Геометрия распространяющегося фотона. Здесь $\vec{n}-$ нормальный вектор. В левой части псевдоскалярный конденсат постоянен, правая часть соответствует области с ненулевым градиентом конденсата.

Таким образом, мы получили решения в обоих полупространствах и должны сшить их на границе. С учетом непрерывности векторного поля интегрирование вдоль компоненты $x_{1}$ от $-\varepsilon$ до $\varepsilon$ дает выражения

$$
\begin{aligned}
& \tilde{u}_{0 \rightarrow}^{(L)}=\frac{\omega^{2}}{\omega^{2}-k_{\perp}^{2}} \tilde{u}_{0 \rightarrow}+\frac{\omega k_{3}}{\omega^{2}-k_{\perp}^{2}} \tilde{u}_{3 \rightarrow}+\frac{\omega k_{2}}{\omega^{2}-k_{\perp}^{2}} \tilde{u}_{2 \rightarrow}, \\
& \tilde{u}_{0 \rightarrow}^{( \pm)}=-\frac{k_{\perp}^{2}}{2\left(\omega^{2}-k_{\perp}^{2}\right)} \tilde{u}_{0 \rightarrow}-\frac{\omega k_{3} \mp i k_{2} \sqrt{\omega^{2}-k_{\perp}^{2}}}{2\left(\omega^{2}-k_{\perp}^{2}\right)} \tilde{u}_{3 \rightarrow}-\frac{\omega k_{2} \pm i k_{3} \sqrt{\omega^{2}-k_{\perp}^{2}}}{2\left(\omega^{2}-k_{\perp}^{2}\right)} \tilde{u}_{2 \rightarrow}, \\
& \tilde{u}_{2 \rightarrow}^{(L)}=-\frac{k_{2}^{2}}{\omega^{2}-k_{\perp}^{2}} \tilde{u}_{2 \rightarrow}-\frac{\omega k_{2}}{\omega^{2}-k_{\perp}^{2}} \tilde{u}_{0 \rightarrow}-\frac{k_{2} k_{3}}{\omega^{2}-k_{\perp}^{2}} \tilde{u}_{3 \rightarrow}, \\
& \tilde{u}_{2 \rightarrow}^{( \pm)}=\frac{\omega^{2}-k_{3}^{2}}{2\left(\omega^{2}-k_{\perp}^{2}\right)} \tilde{u}_{2 \rightarrow}+\frac{\omega k_{2} \mp i k_{3} \sqrt{\omega^{2}-k_{\perp}^{2}}}{2\left(\omega^{2}-k_{\perp}^{2}\right)} \tilde{u}_{0 \rightarrow}+\frac{k_{2} k_{3} \mp i \omega \sqrt{\omega^{2}-k_{\perp}^{2}}}{2\left(\omega^{2}-k_{\perp}^{2}\right)} \tilde{u}_{3 \rightarrow}, \\
& \tilde{u}_{3 \rightarrow}^{(L)}=-\frac{k_{3}^{2}}{\omega^{2}-k_{\perp}^{2}} \tilde{u}_{3 \rightarrow-}-\frac{\omega k_{3}}{\omega^{2}-k_{\perp}^{2}} \tilde{u}_{0 \rightarrow-} \frac{k_{2} k_{3}}{\omega^{2}-k_{\perp}^{2}} \tilde{u}_{2 \rightarrow}, \\
& \tilde{u}_{3 \rightarrow}^{( \pm)}=\frac{\omega^{2}-k_{2}^{2}}{2\left(\omega^{2}-k_{\perp}^{2}\right)} \tilde{u}_{3 \rightarrow+}+\frac{\omega k_{3} \pm i k_{2} \sqrt{\omega^{2}-k_{\perp}^{2}}}{2\left(\omega^{2}-k_{\perp}^{2}\right)} \tilde{u}_{0 \rightarrow}+\frac{k_{2} k_{3} \pm i \omega \sqrt{\omega^{2}-k_{\perp}^{2}}}{2\left(\omega^{2}-k_{\perp}^{2}\right)} \tilde{u}_{2 \rightarrow},
\end{aligned}
$$

где $\tilde{u}_{\nu \rightarrow}=\sum_{A=L, \pm} \tilde{u}_{\nu \rightarrow}^{(A)}$. Каждая компонента обладает своим коэффициентом прохождения [26]

$$
\tilde{v}_{\nu A \rightarrow}=\frac{2 k_{1}^{0}}{k_{1}^{0}+k_{1 A}^{\mathrm{CS}}} \tilde{u}_{\nu \rightarrow}^{(A)} .
$$

Используя выражения (14)-(17), находим амплитуду прошедших волн и записываем коэффициент прохождения как

$$
\mathfrak{T}^{ \pm}=\frac{1}{1+\sqrt{1 \mp \zeta / k_{1}^{0}}} .
$$

Отметим, что продольная поляризация (если она имеет место для ненулевой эффективной массы фотона) не чувствует границы.

На рис. 2 направлению падающих фотонов соответствует угол $\beta$. После прохождения границы угол распространения изменяется на угол $\alpha$ в соответствии с поляризацией фотона. Разложим $\vec{k}: \vec{k}=k_{n} \vec{n}+k_{\perp} \vec{\tau}$. Из статьи [21] известно, что $k_{\perp}$ 


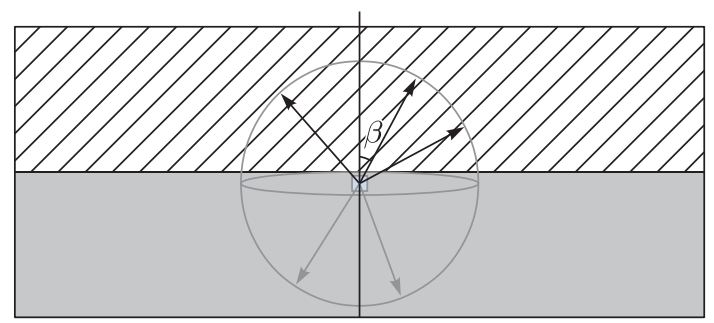

Рис. 3. Излучение малого объема на границе.

остается неизменным при пересечении границы. Это означает, что траектория частицы и нормальный вектор лежат в одной плоскости. Однако $k_{n}$ меняется. Введем новую переменную $k_{n}^{\mathrm{CS}}$ для описания нормальной компоненты $\vec{k}$ после пересечения границы. Для $x_{1}<0$ видно, что

$$
\cos \beta=\frac{k_{1}^{0}}{\omega} .
$$

Мы заинтересованы в нахождении общего потока исходящих фотонов из области 1 в область 2 на рис. 1. Чтобы его найти, рассмотрим малый объем около границы. Предположим, что он равномерно излучает во всех направлениях. Для нас важен поток, идущий наружу, поэтому мы интересуемся только верхней полусферой на рис. 3 .

Допустим, что малый объем излучает с плотностью энергии излучения $N_{\Omega}^{\omega}$ на единицу телесного угла. В данном разделе мы пренебрегаем массой фотона для получения количественных результатов. Для нахождения общей светимости нужно проинтегрировать плотность излучения по телесному углу, частоте и поверхности слоя. Однако последнее интегрирование будет точно таким же, как и в случае отсутствия псевдоскалярного конденсата:

$$
L \propto \int_{0}^{\infty} d \omega N_{\Omega}^{\omega} \int_{\max \left(\frac{\pi}{2}, \arccos \frac{\zeta}{\omega}\right)}^{\pi / 2} d \beta \mathfrak{T}^{+}(\beta, \omega, \zeta)+\int_{0}^{\infty} d \omega N_{\Omega}^{\omega} \int_{0}^{\pi / 2} d \beta \mathfrak{T}^{-}(\beta, \omega, \zeta) .
$$

В данном выражении интегрирование по углам в первом члене начинается со значения $\cos \beta=\zeta / \omega$. Это значение основано на кинематических соображениях для положительной поляризации. Из (18) видно, что для $\mathfrak{T}^{+}$в знаменателе получается отрицательное число под корнем для $\zeta>k_{1}$. Физически это означает, что фотонам с $k_{1}<\zeta$ кинематически запрещено переходить в фотоны с положительной поляризацией в области с линейно меняющейся плотностью псевдоскалярного поля. Для отрицательной поляризации такого ограничения нет, в формуле (6) $k_{1-}$ положительно для всех значений $\zeta$ и, как следствие, никаких специальных пределов интегрирования для второго члена нет.

Предположим, что область находится в тепловом равновесии с температурой $T$ и излучает с плотностью энергии излучения $N_{\Omega}^{\omega} \propto \omega^{3} /\left(e^{\omega / T}-1\right)$. Сравним значения светимости для случаев с эффектами области с нарушением пространственной четности и без них. В случае без подобных эффектов светимость

$$
L_{0} \propto 2 \int_{0}^{\infty} d \omega N_{\Omega}^{\omega} \int_{0}^{\pi / 2} d \beta
$$




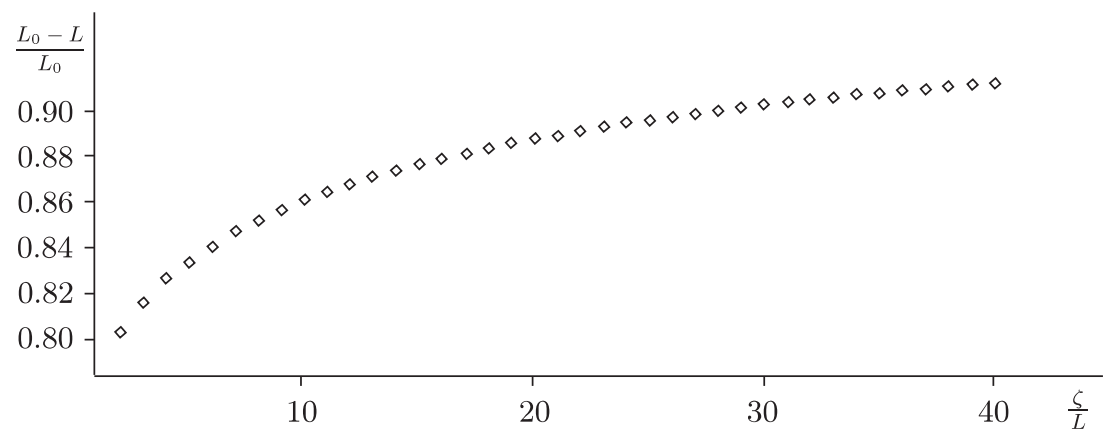

Рис. 4. Относительная разница между светимостью в случае наличия и отсутствия псевдоскалярного конденсата.

где множитель 2 обозначает две обычные фотонные поляризации. На рис. 4 мы приводим график, демонстрирующий влияние границы на светимость.

Из рис. 4 видно, что влияние более чем значительно. Для больших значений $\zeta$ (в сравнении с температурой) большинство фотонов отражаются от границы, тем самым уменьшая количество энергии, выходящей через границу. Также следует обратить внимание на то, что из-за граничных эффектов спектр излучения отличается от спектра черного тела с температурой $T$.

4.2. Выход из граничного слоя. После выхода из первой области фотоны оказываются в области, в которой имеет место электродинамика МЧС. Чтобы проникнуть в следующий домен, фотоны должны пройти через еще одну границу. Она соответствует границе между областями 2 и 3 на рис. 1. Проделаем те же шаги, что и в предыдущем пункте. Рис. 2 и рис. 3 по-прежнему применимы, только области необходимо поменять местами. В нашем случае

$$
\frac{k_{n}^{\mathrm{CS}}}{k_{\perp}}=\operatorname{ctg} \alpha, \quad \frac{k_{n}}{k_{\perp}}=\operatorname{ctg} \beta .
$$

Более того, известно, что для пространственного вектора ЧС имеют место две поперечные поляризации с дисперсионными соотношениями

$$
k_{n \pm}^{\mathrm{CS}}=\sqrt{\omega^{2}-k_{\perp}^{2} \mp \zeta \sqrt{\omega^{2}-k_{\perp}^{2}}}
$$

или, поскольку мы рассматриваем фотон и $k_{n}=\sqrt{\omega^{2}-k_{\perp}^{2}}$,

$$
k_{n \pm}^{\mathrm{CS}}=\sqrt{k_{n}^{2} \mp \zeta k_{n}} .
$$

Используя результат, полученный в статье [21], мы можем найти коэффициент прохождения для каждой из поляризаций:

$$
\mathfrak{T}^{A}=\frac{2 k_{1 A}^{\mathrm{CS}}}{k_{1 A}^{\mathrm{CS}}+k_{1}^{0}} .
$$


Мы заинтересованы только в поперечных поляризациях (продольная поляризация, напомним, не замечает границы), и коэффициент прохождения можно записать как

$$
\mathfrak{T} \pm=\frac{2 k_{n \pm}^{\mathrm{CS}}}{k_{n \pm}^{\mathrm{CS}}+k_{n}}=\frac{2 \operatorname{ctg} \alpha}{\operatorname{ctg} \alpha+\operatorname{ctg} \beta} .
$$

Для наших целей необходимо выразить $\mathfrak{T}^{ \pm}$как функцию от $\beta$, поэтому выразим $\alpha$ через $\beta$. Для постоянного $a(x)$ мы имеем $\omega=|\vec{k}|$, и можно использовать равенство $k_{n}=\omega \cos \alpha$. Поэтому из (22) получаем, что

$$
\operatorname{ctg} \alpha=\operatorname{ctg} \beta \frac{\omega \cos \alpha}{\sqrt{\omega^{2} \cos ^{2} \alpha \mp \zeta \omega \cos \alpha}},
$$

где, как и ранее, знак Ғ принимает определенное значение для соответствующей поляризации.

Решая данное уравнение, легко найти выражение для $\operatorname{ctg} \beta$ для разных поляризаций. Выражение для $\mathfrak{T}^{ \pm}$принимает вид

$$
\mathfrak{T}^{ \pm}(\beta, \zeta, \omega)=\frac{2 \operatorname{ctg} \beta}{\operatorname{ctg} \beta+\frac{ \pm \zeta+\sqrt{\zeta^{2}+4 \omega^{2} \operatorname{ctg}^{2} \beta\left(1+\operatorname{ctg}^{2} \beta\right)}}{\sqrt{4 \omega^{2}\left(1+\operatorname{ctg}^{2} \beta\right)-2 \zeta^{2} \mp 2 \zeta \sqrt{\zeta^{2}+4 \omega^{2} \operatorname{ctg}^{2} \beta\left(1+\operatorname{ctg}^{2} \beta\right)}}}} .
$$

Используя эту формулу, для любого угла $\beta$ можно найти долю фотонов, которым удается пересечь границу $x_{+}$и попасть в область с постоянным $a$. Как и в предыдущем пункте, мы рассматриваем малый объем, который излучает с плотностью энергии $N_{\Omega}^{\omega}$ на единицу телесного угла, и поэтому выражение для полной светимости имеет вид

$$
L \propto \int_{\zeta}^{\infty} d \omega N_{\Omega}^{\omega} \int_{0}^{\pi / 2} d \beta \mathfrak{T}^{+}(\beta, \omega, \zeta)+\int_{0}^{\infty} d \omega N_{\Omega}^{\omega} \int_{0}^{\pi / 2} d \beta \mathfrak{T}^{-}(\beta, \omega, \zeta) .
$$

Следует обратить внимание на области интегрирования обоих членов. Из выражения (22) легко заметить, что отрицательная поляризация не накладывает кинематических условий на фотон. А у фотона с положительной поляризацией $\omega$ не может оказаться меньше $\zeta$.

Как и ранее, для описания качественного эффекта наличия псевдоскалярного конденсата предположим, что среда имеет температуру $T$. Легко заметить, что для $\zeta>T$ наблюдается значительный эффект подавления исходящей энергии, что должно приводить к замедлению процесса радиационного остывания внутренних доменов (область 1 на рис. 1).

Учитывая обе границы, которые должны пройти фотоны для выхода из внутреннего домена, мы получаем еще большее подавление. Если пренебречь теплоемкостью оболочки (область 2 на рис. 1), можно построить график (см. рис. 6), показывающий совместный эффект двух границ внутреннего домена. Если внутренний домен является более горячей областью, чем окружающая среда, описываемый нами эффект действительно должен приводить к замедлению процесса остывания. Однако надо отметить, что в плотных звездах радиационное охлаждение не является доминирующим процессом охлаждения, поэтому такого сильного эффекта, как мы можем видеть на графиках, в реальных системах ожидать не следует. 


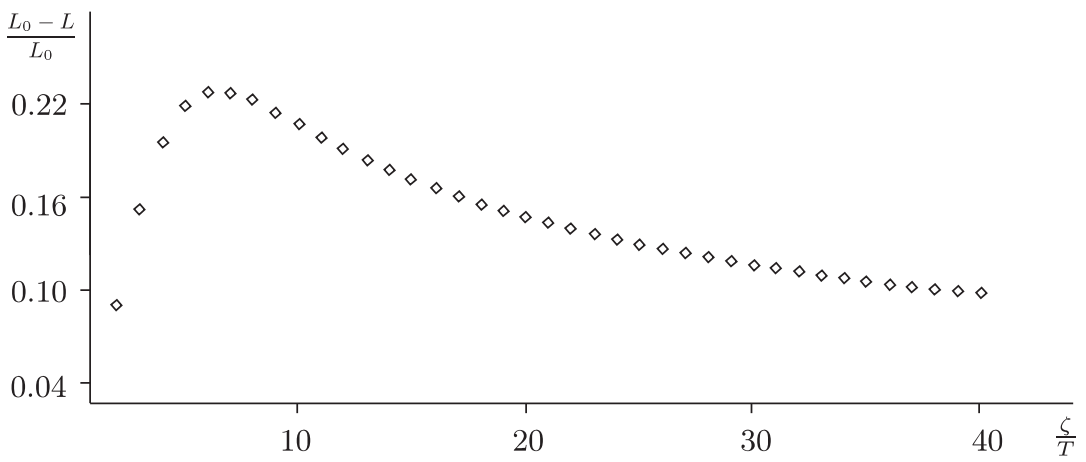

Рис. 5. Относительная разница между светимостью при наличии и отсутствии псевдоскалярного конденсата (случай выхода из среды с нарушенной четностью).

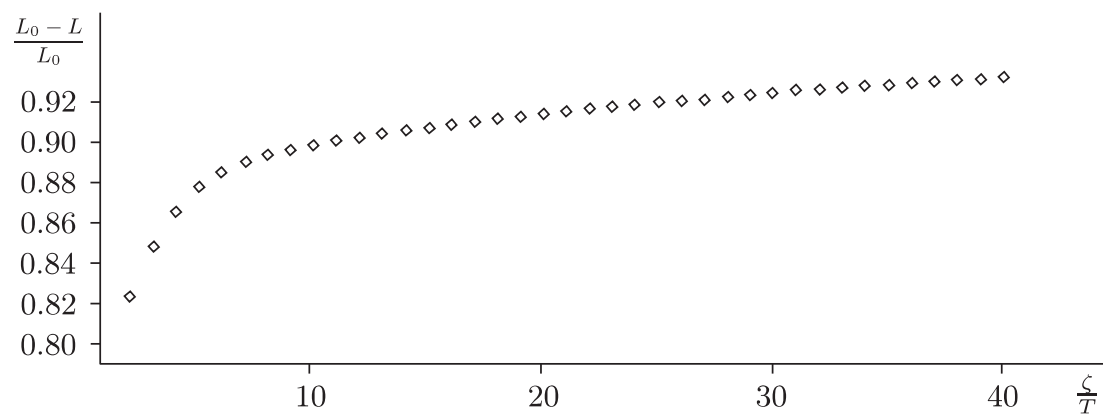

Рис. 6. Относительная разница между светимостью с учетом двух границ.

\section{5. ФЕРМИОННЫЙ РАСПАД В ПРИСУТСТВИИ ПСЕВДОСКАЛЯРНОГО ГРАДИЕНТА}

Фермионы, проходящие через границы доменов, частично отражаются, как и фотоны. Однако поскольку $b \ll m$, подобные эффекты не так значительны. Более важным явлением в данном случае является нестабильность фермионов в области с нарушением пространственной четности. А именно, фермион (это может быть и электрон, и нейтрон) с большей эффективной поперечной массой $m_{+}=\sqrt{p_{1}^{2}+m^{2}}+b$ может распасться на фермион с меньшей эффективной поперечной массой, равной $m_{-}=\sqrt{q_{1}^{2}+m^{2}}-b$, излучив при этом фотон (в случае электрона) или нейтральный виртуальный пион (если речь идет о нейтроне), который сразу распадется на два фотона. Поскольку для обоих случаев применим один и тот же формализм, в данной работе мы рассмотрим только случай испускания фотона электроном в среде с нарушением пространственной четности, обладающей температурой $T$ и химическим потенциалом $\mu$. В этом случае испускаемый фотон обладает поперечной массой $m_{\gamma}=\sqrt{k_{1}^{2}+m_{\star}^{2}+\zeta^{2} / 4} \pm \zeta / 2$, где $m_{\star}$ - эффективная масса фотона в плазме [27], [28]. При очень высоких температурах и плотностях (и значениях химического потенциала $\mu$ ) эффективная масса фотона [27] $m_{\star}^{2} \simeq e^{2}\left(T^{2} / 3+\mu^{2} / \pi^{2}\right) / 3$ возникает как в продольном тензоре поляризации ("плазмон”), так и в поперечном. При этом эти массы 
при нулевых волновых векторах совпадают по величине. Они являются источником превращения фотона в нейтрино и антинейтрино [29].

Для простоты вычислений ограничимся пространственным случаем для вектора $b_{\mu}=(0, b, 0,0)$ и выберем $b>0$. Мы также можем путем выбора координат перейти к системе отсчета, в которой у распадающегося электрона нет поперечных компонент импульса (ведь сокращенный вариант симметрии Лоренца $O(1,2)$ остается на гиперплоскости $(0,2,3))$. В таком случае возможен распад электрона с поперечной массой $m_{+}$и импульсом $p_{\mu}$ на электрон с поперечной массой $m_{-}$и импульсом $q_{\mu}$ и фотон с импульсом $k_{\mu}$ и поперечной массой $m_{\gamma}=\sqrt{k_{1}^{2}+m_{\star}^{2}+\zeta^{2} / 4} \pm \zeta / 2$. Законы сохранения в данном случае приводят нас к следующим выражениям:

$$
\begin{aligned}
b+\sqrt{p_{1}^{2}+m^{2}}= & \sqrt{\vec{q}_{\perp}^{2}+\left(\sqrt{q_{1}^{2}+m^{2}}-b\right)^{2}}+ \\
& +\sqrt{\vec{k}_{\perp}^{2}+\left(\sqrt{k_{1}^{2}+m_{\star}^{2}+\frac{\varepsilon^{2} \zeta^{2}}{4}}+\frac{\varepsilon \zeta}{2}\right)^{2}}, \\
p_{1} & =q_{1}+k_{1}, \quad \vec{q}_{\perp}=-\vec{k}_{\perp},
\end{aligned}
$$

где $\varepsilon=0, \pm 1$ для фотонной продольной и двух поперечных поляризаций соответственно.

Для нахождения порога процесса, в котором электроны могут излучать фотон, для простоты рассмотрим чисто коллинеарный распад, когда $\vec{q}_{\perp}=-\vec{k}_{\perp}=0$ :

$$
b+\sqrt{p_{1}^{2}+m^{2}}=\sqrt{q_{1}^{2}+m^{2}}-b+\sqrt{k_{1}^{2}+m_{\star}^{2}+\frac{\varepsilon^{2} \zeta^{2}}{4}}+\frac{\varepsilon \zeta}{2} .
$$

Более того, рассмотрим систему отсчета, в которой электрон покоится $\left(p_{1}=0\right)$, тогда

$$
2 b+m-\frac{\varepsilon \zeta}{2}=\sqrt{q_{1}^{2}+m^{2}}+\sqrt{q_{1}^{2}+m_{\star}^{2}+\frac{\varepsilon^{2} \zeta^{2}}{4}}>m+\sqrt{m_{\star}^{2}+\frac{\varepsilon^{2} \zeta^{2}}{4}},
$$

откуда можно вывести необходимые неравенства для процесса излучения фотона различных поляризаций:

$$
\begin{aligned}
& L: \quad \varepsilon=0, \quad 2 b \geqslant m_{\star}, \\
& +: \quad \varepsilon=+1, \quad 2 b \sqrt{1-\frac{\zeta}{2 b}} \geqslant m_{\star}, \\
& -: \quad \varepsilon=-1, \quad 2 b \sqrt{1+\frac{\zeta}{2 b}} \geqslant m_{\star} .
\end{aligned}
$$

При очень высоких температурах и плотностях (и значениях химического потенциала $\mu$ ) эффективная масса фотона [27] подавляет переход “+”-электронов в “-"'-электроны в среде с нарушенной четностью.

Переход фермионов из одного вида в другой через испускание фотона может являться процессом поддержания равновесия химического потенциала между уровнями Ферми, приводя к избытку “-”-фермионов в системе. Как мы видели, возможность и интенсивность описываемого процесса очень сильно зависит от соотношения трех параметров $b, \zeta$ и $m_{\star}$. Если из-за величины эффективной массы фотона переход оказывается невозможным, в звезде будут одновременно существовать два моря Ферми. 
Следует обратить внимание на то, что хотя в вакууме все “+"-электроны распались бы на “-"-электроны, в случае звезды такие переходы возможны только для ограниченного числа фермионов, поскольку для остальных фермионов не останется более низких незанятых энергетических уровней. Таким образом, влияние данного эффекта на механизм остывания звезды должно быть совсем небольшим, хотя внезапный выброс фотонов, который может возникнуть при переходе лептонов из одного состояния в другое, может оказаться наблюдаемым сигналом. Наблюдаемость данного процесса зависит от прозрачности внешних слоев звезды для исходящих фотонов. Если же внешние слои непрозрачны, то описываемые процессы будут лишь немного подогревать внутренние области звезды.

\section{6. ВОЗМОЖНЫЕ ОБЛАСТИ ПРИМЕНЕНИЯ}

В настоящей работе мы обсудили несколько интересных эффектов, возможных в области с ненулевым градиентом псевдоскалярного конденсата. Возможность применения полученных результатов для описания реальных физических систем, таких как компактные звезды, зависит от ряда условий и параметров. Некоторые из них можно получить из общих соображений, другие же зависят от конкретного строения астрофизических объектов.

Прежде всего имеет смысл понять порядок величин $b$ и $\zeta$, которые использовались в данной работе. Теоретически аксионный конденсат, соответствующий аксионной теории Печчеи-Квинн, взаимодействует с электронами с константой взаимодействия порядка $y_{\mathrm{e}} \times\left\langle\partial_{x} a(x)\right\rangle / f_{\mathrm{a}}$ где $y_{\mathrm{e}}-$ соответствующий параметр взаимодействия Юкавы, $f_{\mathrm{a}}$ - константа аксионного распада, а скобки $\langle\cdot\rangle$ означают усреднение по конечной области конденсата. При этом $a(x)$ - переменное аксионное поле. Для нейтронов обсуждаемое взаимодействие усилено фактором $m_{\mathrm{n}} / m_{\mathrm{e}} \simeq 20 \div 30$. Основной неизвестной величиной в данном взаимодействии является $\left\langle\partial_{x} a(x)\right\rangle$. Эта величина также определяет параметр $\zeta$.

Пионный конденсат может повлиять на электроны через взаимодействие в $10^{-3}$ раз ме́ньшее, чем с фотонами, связанное с распадом $\pi \rightarrow e^{+} e^{-}[30]$. Что касается нейтронов, влияние переменного пионного конденсата на него значительно больше:

$$
b \simeq g_{\pi \mathrm{NN}} \frac{\left\langle\partial_{x} \pi(x)\right\rangle}{f_{\pi}} .
$$

Поэтому $b$ должно быть больше для процессов с нейтронами.

Для вырожденных электронов оценки энергии Ферми в белых карликах дают величину $0.10 \mathrm{MэВ,} \mathrm{а} \mathrm{для} \mathrm{типичной} \mathrm{нейтронной} \mathrm{звезды} \mathrm{-} 50$ МэВ. В обоих случаях $\epsilon_{\mathrm{F}} \ll m$ и, соответственно, нерелятивистское приближение, которым мы пользовались, корректно.

Пожалуй, самый явный эффект, обсуждаемый в данной работе, - это расщепление моря Ферми на два отдельных набора, соответствующих различным “типам" фермионов, для которых выполняются различные дисперсионные соотношения. В нерелятивистском пределе два уровня Ферми должны быть разделены энергией в $2 b$. При большом гравитационном давлении два уровня будут стремиться перейти в состояние равновесия, при этом произойдет излучение фотонов с характерной частотой $\sim b$.

Возможные последствия эффекта отражения от границ для фотонов оценить сложнее. Излучение, исходящее из внутренних слоев звезды, очень долго добирается до поверхности. К примеру, для Солнца временной масштаб диффузии составляет $\sim 10^{5}$ лет [31]. Нейтронные звезды - гораздо более плотные объекты, чем 
Солнце $\left(\rho \sim \rho_{0}\right)$, так что длина свободного пробега фотона очень мала (описание строения нейтронных звезд можно найти в книге [32]) и принято считать, что после начальной стадии остывания, в которой доминирующую роль играет излучение нейтрино, в течение большей части времени жизни звезды теплообмен происходит за счет тепловой диффузии вырожденного нейтронного газа, а радиационное охлаждение может быть эффективно только во внешних слоях звезды (коре и атмосфере). Внутренняя температура примерно постоянна благодаря высокой теплопроводности нейтронов, возникающей из-за их большой длины свободного пробега, что в свою очередь является следствием свойств вырожденного газа Ферми. Скорость остывания нейтронных звезд зависит в большой степени от прозрачности внешних слоев [32]. Следовательно, механизмы, описываемые в настоящей работе, могут влиять на остывание звезды, если ненулевой градиент псевдоскалярного конденсата располагается недалеко от коры звезды. Все наши рассуждения, конечно, основаны на правильности представления стандартного механизма остывания подобных звезд.

Белые карлики имеют в своем составе вырожденный релятивистский электронный газ, и механизм их остывания в квазистабильном состоянии похож на обсуждаемый выше [33]. Электрон-электронное взаимодействие не играет в данном случае важнейшей роли по тем же причинам, что и для нейтронов в случае нейтронной звезды, и теплообмен происходит в основном за счет электрон-ионных взаимодействий (ионы в данном случае подчиняются классической физике, поскольку температура меньше их химического потенциала). Более того, наибольшее количество тепла, передаваемого внутри белых карликов, переносится положительными ионами (в классической термодинамике для ионов $C_{V}^{\mathrm{i}} \sim 3 N_{\mathrm{i}} / 2$, тогда как для вырожденного электронного газа $\left.C_{V}^{\mathrm{e}} \sim N_{\mathrm{e}} T / \epsilon_{\mathrm{F}} \ll C_{V}^{\mathrm{i}}\right)$. Фотоны с энергиями порядка нескольких КэВ слабо взаимодействуют с электронами из-за их вырожденности, энергия Ферми для них имеет порядок 0.1-0.2 МэВ, но они могут беспрепятственно взаимодействовать с ионами, и радиационное охлаждение имеет большое значение для внешних слоев звезды.

В любом случае описываемые в настоящей работе процессы, связанные с фотонами, работают на замедление процесса остывания. Мы также отметили подобные эффекты для фермионов, но из-за фермионной массы они должны быть незначительными.

\section{7. ЗАКЛЮЧЕНИЕ}

В данной работе мы рассмотрели влияние псевдоскалярного конденсата на свойства фотонов и фермионов в звездной материи. Несмотря на то что мы брали за основу общепринятые данные о звездах, описанные в работе эффекты получены на основании ряда предположений. В частности, мы строили нашу модель исходя из того, что четность может спонтанно нарушаться из-за высокой плотности или присутствия аксионного конденсата. Мы предположили, что внутри звезды должно происходить образование доменов с подобным нарушением пространственной четности, только в этом случае описываемые процессы дадут вклад в физику внутренних слоев звезды. Это предположение кажется обоснованным, поскольку в случае нарушения пространственной четности должна существовать хотя бы одна область, окруженная пространством без псевдоскалярного конденсата. Для описания процессов отражения и прохождения мы использовали предположение о характерных размерах доменов и переходных слоев между ними, считая, что эти размеры должны быть много больше характерной длины волны фотонов и электронов. 
Появление псевдоскалярного конденсата в ядерной материи при высокой барионной плотности до сих пор не было экспериментально обнаружено, но теоретически было предсказано в работе [8]. Кроме того, в последнее время стали появляться работы, обсуждающие возможность существования аксионного конденсата Бозе-Эйнштейна с радиусом от 10 до $10^{5}$ км. Если это действительно так, подобные конденсаты смогут играть значительную роль в физике звезд.

Таким образом, если наши предположения верны, процесс стабилизации звезды и процесс остывания зависят в той или иной степени от наличия градиента псевдоскалярного конденсата в звездной материи. Следует обратить внимание на то, что описываемые в нашей работе процессы зависят только от параметров, определяемых распределением пионного/аксионного конденсата. Поэтому для получения численных расчетов (например, для процесса остывания) нам необходимо знать характерные размеры доменов, их распределения и параметры конденсата.

Интересные возможности для обнаружения эффектов нарушения пространственной четности могут открыться при наложении сильных магнитных полей в присутствии температуры и плотности фермионов [34].

В заключение еще раз отметим эффекты, предсказанные в нашей работе. Фермионные и фотонные спектры в среде искажаются. За счет этого происходит появление нетривиальных граничных процессов: часть частиц, падающих на границу области с нарушенной четностью, отражается. Это в свою очередь может привести к замедлению остывания внутренних слоев звезды. Эффекты отражения свойственны и фотонам, и фермионам, однако для фермионов они не так значительны. Более важным эффектом для половины из них является возможность распада с излучением фотона (возможность распада фотонов тоже существует и была описана ранее [35]). Но лишь небольшая часть может распасться из-за принципа Паули. Различие двух морей Ферми, соответствующих двум фермионным типам в среде, может привести к резкому переходу части фермионов в другое состояние за счет гравитационного сжатия. Подобный процесс будет сопровождаться выбросом фотонов с определенной энергией. Конечно, все указанные эффекты прежде всего зависят от величины параметров, описывающих нарушение пространственной четности.

В ближайшем будущем несколько новых астрофизических экспериментов [36] могут предоставить новую информацию о нейтронных звездах. В частности, космическая миссия NICER [37], которая должна начаться в конце 2017 г., скорее всего позволит нам открыть десятки тысяч нейтронных звезд, поможет понять внутреннее строение этих объектов и, возможно, найти следы нарушения четности из-за псевдоскалярного конденсата.

\section{Решение уравнений МЧС}

ПРИЛОЖЕНИЕ А

Напомним вид так называемых киральных векторов поляризации для векторного поля МЧС. Отправная точка - это симметричный тензор второго ранга, проектор на плоскость, поперечную векторам $k_{\mu}, \zeta_{\nu}[21]$ :

$$
S_{\lambda}^{\nu} \equiv \varepsilon^{\mu \nu \alpha \beta} \zeta_{\alpha} k_{\beta} \varepsilon_{\mu \lambda \rho \sigma} \zeta^{\rho} k^{\sigma}=\delta_{\lambda}^{\nu} \mathrm{D}+k^{\nu} k_{\lambda} \zeta^{2}+\zeta^{\nu} \zeta_{\lambda} k^{2}-\zeta \cdot k\left(\zeta_{\lambda} k^{\nu}+\zeta^{\nu} k_{\lambda}\right),
$$

где

$$
\mathrm{D} \equiv(\zeta \cdot k)^{2}-\zeta^{2} k^{2}=\frac{1}{2} S^{\nu}{ }_{\nu}
$$


Используя последнее равенство, можно получить следующее соотношение:

$$
S^{\mu \lambda} \varepsilon_{\lambda \nu \alpha \beta} \zeta^{\alpha} k^{\beta}=\mathrm{D} \varepsilon_{\nu \alpha \beta}^{\mu} \zeta^{\alpha} k \beta .
$$

Для получения векторов поляризации введем два ортонормированных одномерных эрмитовых проектора:

$$
\boldsymbol{\pi}_{ \pm}^{\mu \nu} \equiv \frac{S^{\mu \nu}}{2 \mathrm{D}} \pm \frac{i}{2} \varepsilon^{\mu \nu \alpha \beta} \zeta_{\alpha} k_{\beta} \mathrm{D}^{-1 / 2}=\left(\boldsymbol{\pi}_{ \pm}^{\nu \mu}\right)^{*}=\left(\boldsymbol{\pi}_{\mp}^{\mu \nu}\right)^{*}, \quad \mathrm{D}>0 .
$$

Два киральных вектора поляризации для векторного поля МЧС можно построить из тетрад $\epsilon_{\nu}$, состоящих из постоянных значений:

$$
\varepsilon_{ \pm}^{\mu}(k)=\pi_{ \pm}^{\mu \lambda} \epsilon_{\lambda}
$$

Их свойства описаны в работе [21].

Теперь, чтобы получить нормальные моды распространения поля МЧС, введем кинетическую эрмитову $(4 \times 4)$-матрицу $\mathbb{K}$ с элементами

$$
K_{\lambda \nu} \equiv g_{\lambda \nu}\left(k^{2}-m^{2}\right)+i \varepsilon_{\lambda \nu \alpha \beta} \zeta^{\alpha} k^{\beta}, \quad K_{\lambda \nu}=K_{\nu \lambda}^{*}
$$

Общее решение уравнений свободного поля (4) при $x_{1}>0$ можно найти, используя (А.3) и (А.4):

$$
K^{\mu}{ }_{\nu} \varepsilon_{ \pm}^{\nu}(k)=\left[\delta^{\mu}{ }_{\nu}\left(k^{2}-m^{2}\right)+\sqrt{\mathrm{D}}\left(\boldsymbol{\pi}_{+\nu}^{\mu}-\boldsymbol{\pi}_{-\nu}^{\mu}\right)\right] \varepsilon_{ \pm}^{\mu}(k)=\left(k^{2}-m^{2} \pm \sqrt{\mathrm{D}}\right) \varepsilon_{ \pm}^{\mu}(k) .
$$

ПРИЛОЖКНИЕ Б

\section{Решения модифицированного уравнения Дирака}

Модифицированное уравнение Дирака в импульсном пространстве имеет вид

$$
\left(\gamma^{\mu} p_{\mu}-m-\gamma^{\mu} b_{\mu} \gamma^{5}\right) \psi=0
$$

Удобно использовать следующие представления:

$$
\gamma^{0} \gamma^{1} \gamma^{5}=-i \gamma^{0} \gamma^{1} \gamma^{0} \gamma^{1} \gamma^{2} \gamma^{3}=-i \gamma^{2} \gamma^{3}=-\left(\begin{array}{cc}
\sigma_{1} & 0 \\
0 & \sigma_{1}
\end{array}\right) \equiv-\hat{\sigma}_{1} .
$$

Используя их и умножая первое уравнение на $\gamma^{0}$, получаем уравнение

$$
\left(\gamma^{0} \gamma^{\mu} p_{\mu}-\gamma^{0} m+b \hat{\sigma}_{1}\right) \psi=0
$$

Введем два проектора

$$
\mathbf{P}_{ \pm} \equiv \frac{I \pm \hat{\sigma}_{1}}{2}, \quad \psi_{ \pm}=\mathbf{P}_{ \pm} \psi
$$

Их можно использовать, чтобы упростить (Б.3) с помощью нижеследующих соотношений:

$$
\left[\hat{\sigma}_{1}, \gamma_{0}\right]=0, \quad\left[\hat{\sigma}_{1}, \gamma_{1}\right]=0, \quad\left\{\hat{\sigma}_{1}, \gamma_{2}\right\}=0, \quad\left\{\hat{\sigma}_{1}, \gamma_{3}\right\}=0
$$

а именно, получаем, что

$$
\mathbf{P}_{+}\left(\gamma^{0} \gamma^{\mu} p_{\mu}-\gamma^{0} m+b \hat{\sigma}_{1}\right) \psi=0 \quad \Longleftrightarrow \quad\left(p_{0}-\alpha_{1} p_{1}-\gamma^{0} m+b\right) \psi_{+}-\alpha_{\perp} p_{\perp} \psi_{-}=0
$$


а также уравнение

$$
\left(p_{0}-\alpha_{1} p_{1}-\gamma^{0} m-b\right) \psi_{-}-\alpha_{\perp} p_{\perp} \psi_{+}=0,
$$

где $\alpha_{i}=\gamma^{0} \gamma^{i}$ и $\alpha_{\perp} p_{\perp}=\alpha_{2} p_{2}+\alpha_{3} p_{3}$. Чтобы решить уравнения (Б.6), (Б.7), умножаем (Б.6) на $\alpha_{\perp} p_{\perp}$ и находим, что

$$
\psi_{-}=\frac{\left(\alpha_{\perp} p_{\perp}\right)\left(p_{0}-\alpha_{1} p_{1}-\gamma^{0} m+b\right)}{p_{\perp}^{2}} \psi_{+} .
$$

При помощи этих уравнений получаем равенство

$$
\left[\left(p_{0}-\alpha_{1} p_{1}-\gamma^{0} m-b\right) \frac{\alpha_{\perp} p_{\perp}}{p_{\perp}^{2}}\left(p_{0}-\alpha_{1} p_{1}-\gamma^{0} m+b\right)-\alpha_{\perp} p_{\perp}\right] \psi_{+}=0
$$

и для $\psi_{-}, \psi_{+}$записываем следующее уравнение:

$$
\left(p_{0}^{2}-\mathbf{p}^{2}-m^{2}-b^{2} \pm 2 b\left(\alpha_{1} p_{1}+\gamma^{0} m\right)\right) \psi_{ \pm}=0
$$

Чтобы его решить, выражаем $\psi_{ \pm}$как $\psi_{ \pm}=\left(\begin{array}{c}\phi_{ \pm} \\ \xi_{ \pm}\end{array}\right)$. Используя его, запишем (Б.9) в виде

$$
\begin{aligned}
& \left(p_{0}^{2}-\mathbf{p}^{2}-m^{2}-b^{2} \pm 2 b m\right) \phi_{ \pm} \pm 2 b p_{1} \sigma_{1} \xi_{ \pm}=0 \\
& \left(p_{0}^{2}-\mathbf{p}^{2}-m^{2}-b^{2} \mp 2 b m\right) \xi_{ \pm} \pm 2 b p_{1} \sigma_{1} \phi_{ \pm}=0 .
\end{aligned}
$$

Из уравнений (Б.10) нетрудно получить, что

$$
\left[\left(p_{0}^{2}-\mathbf{p}^{2}-m^{2}-b^{2}\right)^{2}-4 b^{2} m^{2}-4 b^{2} p_{1}^{2}\right] \phi_{ \pm}=0,
$$

откуда следует, что дисперсионный закон имеет вид

$$
p_{0}^{2}=\mathbf{p}^{2}+m^{2}+b^{2} \pm 2 b \sqrt{m^{2}+p_{1}^{2}} .
$$

В итоге поле Дирака $\psi(x)$ можно представить следующим образом:

$$
\psi(x)=\sum_{A= \pm} u_{A}(p) e^{-i \hat{p} \hat{x}+p_{1 A} x_{1}},
$$

где

$$
p_{1 \mp}=\sqrt{p_{0}^{2}-p_{\perp}^{2}+3 b^{2}-m^{2} \pm 2 b \sqrt{p_{0}^{2}-p_{\perp}^{2}+2 b^{2}-2 m^{2}}} .
$$

\section{Список литературы}

[1] S. M. Carroll, G. B. Field, R. Jackiw, "Limits on a Lorentz- and parity-violating modification of electrodynamics", Phys. Rev. D, 41:4 (1990), 1231.

[2] B. I. Abelev, M. M. Aggarwal, Z. Ahammed et al. [STAR Collab.], "Azimuthal chargedparticle correlations and possible local strong parity violation", Phys. Rev. Lett., 103:25 (2009), 251601, 7 pp.; "Observation of charge-dependent azimuthal correlations and possible local strong parity violation in heavy-ion collisions", Phys. Rev. C, 81:5 (2010), 054908, $15 \mathrm{pp}$. 
[3] D. Kharzeev, "Parity violation in hot QCD: why it can happen, and how to look for it", Phys. Lett. B, 633:2-3 (2006), 260-264; D. Kharzeev, A. Zhitnitsky, "Charge separation induced by P-odd bubbles in QCD matter", Nucl. Phys. A, 797:1-2 (2007), 67-79; K. Fukushima, D.E. Kharzeev, H. J. Warringa, "Chiral magnetic effect", Phys. Rev. D, 78:7 (2008), 074033, 14 pp.; D. E. Kharzeev, L. D. McLerran, H. J. Warringa, "The effects of topological charge change in heavy ion collisions: 'Event by event PP and CPCP violation'", Nucl. Phys. A, 803:3-4 (2008), 227-253.

[4] A. B. Migdal, "Pion fields in nuclear matter", Rev. Modern Phys., 50:1 (1978), 107-172; D. Bailin, A. Love, "Superfluidity and superconductivity in relativistic fermion systems", Phys. Rep., 107:6 (1984), 325-385; Т. Эриксон, В. Вайзе, Пионы и ядра, Наука, М., 1991; C.-H. Lee, "Kaon condensation in dense stellar matter?", Phys. Rep., 275:5-6 (1996), 255-341; M. Prakash, I. Bombaci, M. Prakash, P. J. Ellis, J. M. Lattimer, R. Knorren, "Composition and structure of protoneutron stars", Phys. Rep., 280:1 (1997), 1-77.

[5] R. Arnaldi, R. Averbeck, K. Banicz et al. [NA60 Collab.], "First measurement of the $\rho$ spectral function in high-energy nuclear collisions", Phys. Rev. Lett., 96:16 (2006), 162302, 5 pp.

[6] A. Adare, S. Afanasiev, C. Aidala et al. [PHENIX Collab.], "Detailed measurement of the $e^{+} e^{-}$pair continuum in $p+p$ and $\mathrm{Au}+\mathrm{Au}$ collisions at $\sqrt{s_{N N}}=200 \mathrm{GeV}$ and implications for direct photon production", Phys. Rev. C, 81:3 (2010), 034911, 56 pp.

[7] A. A. Andrianov, V. A. Andrianov, D. Espriu, X. Planells, "Dilepton excess from local parity breaking in baryon matter", Phys. Lett. B, 710:1 (2012), 230-235; Abnormal dilepton yield from local parity breaking in heavy-ion collisions, arXiv: 1010.4688v1.

[8] A. A. Andrianov, D. Espriu, "On the possibility of $P$-violation at finite baryon-number densities", Phys. Lett. B, 663:5 (2008), 450-455; A. A. Andrianov, V. A. Andrianov, D. Espriu, "Spontaneous P-violation in QCD in extreme conditions", Phys. Lett. B, 678:4 (2009), 416-421; "Spontaneous parity violation under extreme conditions: an effective lagrangian analysis", Eur. Phys. J. C, 74:6 (2014), 2932, 23 pp.

[9] G. D. Moore, M. Tassler, "The sphaleron rate in $\mathrm{SU}(N)$ gauge theory", JHEP, 02 (2011), 105.

[10] D. E. Kharzeev, "Topology, magnetic field, and strongly interacting matter", Ann. Rev. Nucl. Part. Sci., 65 (2015), 193-214.

[11] A. A. Andrianov, R. Soldati, "Lorentz symmetry breaking in Abelian vector-field models with Wess-Zumino interaction", Phys. Rev. D, 51:10 (1995), 5961-5964.

[12] V.A. Kostelecký, M. Mewes, "Signals for Lorentz violation in electrodynamics", Phys. Rev. D, 66:5 (2002), 056005, 24 pp.

[13] G. M. Shore, "Quantum gravitational optics", Contemp. Phys., 44:6 (2003), 503-521.

[14] N. Arkani-Hamed, H. C. Cheng, M. A. Luty, S. Mukohyama, "Ghost condensation and a consistent infrared modification of gravity", JHEP, 05 (2004), 074, 36 pp.; H. C. Cheng, M. A. Luty, S. Mukohyama, J. Thaler, "Spontaneous Lorentz breaking at high energies", JHEP, 05 (2006), 076, 50 pp.

[15] F.E. Schunck, E.W. Mielke, "General relativistic boson stars", Class. Quantum Grav., 20:20 (2003), R301-R356.

[16] J. Eby, P. Suranyi, C. Vaz, L.C.R. Wijewardhana, "Axion stars in the infrared limit", JHEP, 03 (2015), 080, 14 pp., arXiv: 1412.3430v2; A. Iwazaki, "Axion stars and fast radio bursts", Phys. Rev. D, 91:2 (2015), 023008, 4 pp.; Fast radio bursts from axion stars, arXiv: 1412.7825; P.-H. Chavanis, "Relativistic self-gravitating Bose-Einstein condensates and cold baryons with a stiff equation of state", Eur. Phys. J. Plus, 130 (2015), 181, arXiv: 1412.0005 .

[17] T. Muto, T. Tatsumi, "On the cooling of neutron stars under the neutral pion condensed phase", Prog. Theor. Phys., 80:1 (1988), 28-33.

[18] B. Mohanty, J. Serreau, "Disoriented chiral condensate: theory and experiment", Phys. Rep., 414:6 (2005), 263-358, arXiv: hep-ph/0504154. 
[19] A. A. Andrianov, P. Giacconi, R. Soldati, "Lorentz and CPT violations from Chern-Simons modifications of QED", JHEP, 02 (2002), 030.

[20] V.Ch. Zhukovsky, A.E. Lobanov, E. M. Murchikova, "Radiative effects in the standard model extension", Phys. Rev. D, 73:6 (2006), 065016, 8 pp.

[21] A. A. Andrianov, S. S. Kolevatov, R. Soldati, "Propagation of photons and massive vector mesons between a parity breaking medium and vacuum", JHEP, 11 (2011), 007.

[22] A.E. Shabad, V.V. Usov, "Real and virtual photons in an external constant electromagnetic field of most general form", Phys. Rev. D, 81:12 (2010), 125008, 15 pp.; "Effective Lagrangian in nonlinear electrodynamics and its properties of causality and unitarity", Phys. Rev. D, 83:10 (2011), 105006, 16 pp.

[23] А. Е. Шабад, "Сложение скоростей и замкнутый временной цикл в теориях с нарушенной лоренц-инвариантностью”, ТМФ, 187:3 (2016), 421-432, arXiv: 1511.08785.

[24] A. A. Andrianov, V.A. Andrianov, D. Espriu, S.S. Kolevatov, "Stellar matter with pseudoscalar condensates", Eur. Phys. J. C, 76 (2016), 169, 13 pp., arXiv: 1507.07087.

[25] V. A. Kostelecký, R. Lehnert, "Stability, causality and Lorentz and CPT violation", Phys. Rev. D, 63:6 (2001), 065008, 19 pp.

[26] А.А. Андрианов, С.С. Колеватов, "Прохождение векторными частицами границы между средой с нарушением пространственной четности и вакуумом", ТМФ, 175:3 (2013), 357-369, arXiv: 1212.5723.

[27] J. I. Kapusta, C. Gale, Finite-Temperature Field Theory: Principles and Applications, Cambridge Univ. Press, Cambridge, 2006.

[28] Е. С. Фрадкин, "Метод функций Грина в теории квантованных полей и в квантовой статистике", Тр. ФИАН, 29 (1965), 7-138.

[29] E. Braaten, D. Segel, "Neutrino energy loss from the plasma process at all temperatures and densities", Phys. Rev. D, 48:4 (1993), 1478-1491.

[30] K. A. Olive, K. Agashe, C. Amsler et al. [Particle Data Group], "Review of particle physics", Chinese Phys. C, 38:9 (2014), 090001.

[31] R. Mitalas, K. Sills, "On the photon diffusion time scale for the sun", Astrophys. J., 401:2 (1992), 759-760.

[32] P. Haensel, A. Y. Potekhin, D. G. Yakovlev, Neutron Stars 1: Equation of State and Structure, Springer, New York, 2007.

[33] E. Schatzman, White Dwarfs, North-Holland, Amsterdam, 1958; D. G. Yakovlev, D. A. Shalybkov, "Degenerate cores of white dwarfs and envelopes of neutron stars: thermodynamics and plasma screening in thermonuclear reactions", Sov. Sci. Rev. Ser. E, 7 (1989), 311-386.

[34] А.Е. Шабад, "Поляризация вакуума и квантово-релятивистского газа во внешнем поле", Тр. ФИАН, 192 (1988), 5-192; Н. Pérez Rojas, А. E. Shabad, "Polarization of relativistic electron and positron gas in a strong magnetic field. Propagation of electromagnetic waves", Ann. Phys. (N. Y.), 121:1-2 (1979), 432-455.

[35] А. А. Андрианов, Д. Эсприу, С. С. Колеватов, "Поиск нарушения четности в космических лучах", ТМФ, 184:3 (2015), 392-397.

[36] F. Weber, G. A. Contrera, M. G. Orsaria, W. Spinella, O. Zubairi, "Properties of high-density matter in neutron stars", Modern Phys. Lett. A, 29:23 (2014), 1430022, 13 pp.

[37] Z. Arzoumanian, C. Gendreau, C. L. Baker et al., "The neutron star interior composition explorer (NICER): mission definition", Space Telescopes and Instrumentation 2014: Ultraviolet to Gamma Ray, Proceedings of SPIE, 9144, eds. T. Takahashi, J.-W. A. den Herder, M. Bautz, SPIE, Bellingham, WA, 2014, 914420. 